\title{
Hydrogen Bond Induced Nonmonotonic Composition Behavior of the Glass Transition in Aqueous Binary Mixtures
}

\author{
Johan Sjöström, ${ }^{+, *}$ Johan Mattsson, ${ }^{+, \neq, *}$ Rikard Bergman, ${ }^{\dagger}$ and Jan Swenson ${ }^{+}$ \\ ${ }^{\dagger}$ Department of Applied Physics, Chalmers University of Technology, SE-412 96, Göteborg, Sweden \\ ${ }^{\ddagger}$ School of Physics \& Astronomy, University of Leeds, Leeds, LS2 9JT, U.K.
}

ABSTRACT: The glass transition temperature, $T_{\mathrm{g}}$, of a binary mixture commonly varies monotonically between the $T_{\mathrm{g}} \mathrm{s}$ of its two components. However, mixtures of strongly associating liquids can instead exhibit a nonmonotonic $T_{\mathrm{g}}$ variation. The origins of such nonideal mixing behavior have often been correlated with composition dependent structural variations. For binary mixtures between a hydrogen- $(\mathrm{H}-)$ bonded liquid and water, however, such behavior is generally not well understood. The ubiquity and importance of aqueous mixtures both in nature and in man-made applications stresses the needed for a better understanding. We here demonstrate nonmonotonic $T_{\mathrm{g}}$ variations in binary mixtures of $n$-propylene glycol monomethyl ethers ( $n$ PGMEs) and water, where the composition dependent $T_{\mathrm{g}}$ show maxima within an intermediate composition range. We show that these $T_{\mathrm{g}}$ maxima correspond to crossovers in the composition dependence of the step amplitude in the isobaric heat capacity at $T_{\mathrm{g}}$. We further demonstrate that the observed effects are

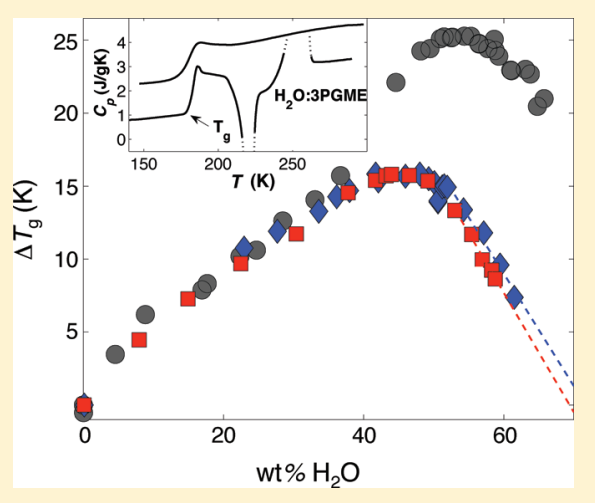
caused by H-bond interactions involving the $n$ PGME hydroxyl group. We can account for our obervations using a simple model based on two effects due to the added water: (i) an H-bond induced formation of effective relaxing entities and (ii) a plasticizing effect at high water contents.

$\mathrm{T}$ he macroscopic properties of a liquid are closely linked to its structural $\alpha$ relaxation. As a liquid is cooled into its supercooled regime, its $\alpha$ relaxation undergoes a dramatic slowing down, which remains poorly understood. As the characteristic time scale of the $\alpha$ relaxation exceeds the longest probed experimental time scale, $\sim 100 \mathrm{~s}$, the sample falls out of thermal equilibrium and effectively forms a disordered solid-a glass. The temperature where the liquid leaves equilibrium is called the glass transition temperature, $T_{\mathrm{g}}$, and is an important characteristic of any glass-forming material. For binary mixtures of glassforming liquids, the $T_{\mathrm{g}}$ values normally vary monotonically between the component $T_{\mathrm{g}} \mathrm{s}$ and are often described using the so-called Gordon-Taylor ( $\mathrm{G}-\mathrm{T}$ ) equation, ${ }^{1}$ which assumes ideal mixing and can be derived using free-volume arguments. ${ }^{2}$ Also nonideal mixtures often show monotonic $T_{\mathrm{g}}$ variations and the $\mathrm{G}-\mathrm{T}$ equation can sometimes still qualitatively describe the composition behavior.

In contrast, there are several examples of binary mixtures that show a nonmonotonic behavior. First, these include a few covalently bonded binary structural glasses, ${ }^{3-5}$ such as the binary mixture $\mathrm{As}_{x}-\mathrm{Se}_{1-x}$. This binary glass has a $T_{\mathrm{g}}$ maximum at the composition $x=0.4$, for which a maximum number of As-Se bonds are formed; since As-Se bonds are stronger than either As-As or $\mathrm{Se}-\mathrm{Se}$ bonds the result is a $T_{\mathrm{g}}$ maximum. ${ }^{4}$ Second, nonmonotonic behaviors are observed for $\mathrm{H}$-bonded mixtures of amines $\left(\mathrm{R}-\mathrm{NH}_{2}\right)$ and alcohols, where a $T_{\mathrm{g}}$ maximum is observed for nearly equal molar concentrations; ${ }^{6-9}$ this behavior can be accounted for by the improved H-bonding ability of the mixed state. ${ }^{8,10}$ A third example is the nonmonotonic composition dependence observed for mixtures of deoxycholic acid and tri-Omethyl- $\beta$-cyclodextrin that are mixed and vitrified by mechanical milling. ${ }^{11}$ These observations were also suggested to result from composition dependent structural changes since both components are known to form inclusion compounds. Thus, a common theme for systems where nonmonotonic $T_{\mathrm{g}}$ behaviors occur is the existence of significant composition dependent structural variations.

An important class of binary mixtures consists of an H-bonding liquid and water. Aqueous liquid mixtures are ubiquitous both in nature and in technology, and important examples include the sugar solutions in plants, the cytoplasm of our cells and the cryoprotectants used for protein storage. ${ }^{12}$ Examples of nonmonotonic $T_{\mathrm{g}}$ variations for completely amorphous aqueous systems have to our knowledge only been observed for aqueous butyltripropylammonium chloride (BTPC), ${ }^{13}$ which shows a $T_{\mathrm{g}}$ maximum for intermediate water concentrations. ${ }^{13,14}$ The effect was suggested to be related to the formation of clathrate type water structures induced by the polar end-groups of BTPC, but significant phase separation and thus multiple $T_{\mathrm{g}} \mathrm{s}$ complicate the analysis. To our knowledge, all other reports for single-phase aqueous mixtures show monotonic $T_{\mathrm{g}}$ variations within the composition range where no crystallization is observed.

\footnotetext{
Received: March 14, 2011

Revised: July 10, 2011

Published: July 12, 2011
} 
For water that is mixed with an alcohol, the mixing entropy is often clearly lower than that expected for an ideal mixture. This has traditionally been interpreted as due to solute induced structuring of water. ${ }^{15}$ However, neutron diffraction studies together with empirical potential structure refinement techniques have revealed that the low mixing entropy can instead be rationalized as due to nanoscopic phase segregation. ${ }^{16,17}$ For water-methanol mixtures, support was found for a scenario where water, at low concentrations, form clusters that are strongly coordinated to the hydroxyl groups of the surrounding methanol molecules. ${ }^{16,18}$ The methanol hydroxyl groups act as links between the hydrophobic methyl groups and the water clusters and it was also found that the methyl groups themselves display clustering. The room temperature viscosity of the mixture displays a nonmonotonic concentration dependence with a maximum observed for a water-methanol molar ratio of $\sim 3: 1$ and it has been found that at this composition both the water molecules and the methyl groups form percolating networks. ${ }^{18}$ The "local" water structure in such mixtures was observed to be remarkably similar to that of pure water. Because of interference from crystallization, however, systematic investigations of mixtures of water and monoalcohols have rarely been carried out in the supercooled temperature range. ${ }^{19,20}$

We here address the present lack of understanding of mixed aqueous systems by performing a systematic investigation of the glass transition behavior of mixtures of water and a series of model H-bonded liquids, $n$-propylene glycol monomethyl ethers, $n$ PGMEs, with the structure $\mathrm{CH}_{3}-\left[\mathrm{OCH}_{2} \mathrm{CH}\left(\mathrm{CH}_{3}\right)\right]_{n}-\mathrm{OH}$ where $n=1,2,3$ for the monomer, dimer and trimer, respectively. In aqueous mixtures, all three liquids show nonmonotonic $T_{\mathrm{g}}$ behaviors with maxima at intermediate water fractions; this behavior contrasts the commonly observed monotonic variation between the components $T_{\mathrm{g}} \mathrm{s}$. We demonstrate that the observed behavior is directly related to the presence of hydroxyl endgroups on the $n$ PGME molecules and show that the effect disappears if these are exchanged for non-H-bonding groups. We further find that nonmonotonic $T_{\mathrm{g}}$ behaviors are observed also for mixtures of $n$ PGMEs and several other $\mathrm{H}$-bonded liquids. We are able to account for our observations using a simple model based on the $\mathrm{H}$-bond mediated building up of larger effective structural units upon addition of water, and a water-induced plasticizing effect at high water concentrations.

\section{EXPERIMENTAL SECTION}

All liquids were purchased from Sigma-Aldrich and freezedried before measurements. ${ }^{21}$ We characterize the glass transitions by measuring the calorimetric heat capacity, $C_{p}$, for cooling/ heating rates of $-30 /+10 \mathrm{~K} \mathrm{~min}^{-1}$ using a differential scanning calorimeter (TA Instruments, Q1000). The experimental procedure is described in more detail in ref. ${ }^{22} C_{p}$ exhibits a step at $T_{\mathrm{g}}$ as the structural relaxation time crosses the time-scale set by the cooling/heating rate. Both the $T_{\mathrm{g}}$ values, determined as the onsets of the heat capacity steps upon heating, see the inset of Figure 1 , and the heights of the steps in $C_{p}$ at $T_{g}, \Delta C_{p}$, were determined.

\section{RESULTS}

The results of the calorimetric measurements of water $-n$ PGME mixtures, are shown in Figure 1. On cooling, no sign of crystallization is found for any sample, see inset of Figure 1. On heating, all samples reveal one distinct glass transition. At high water

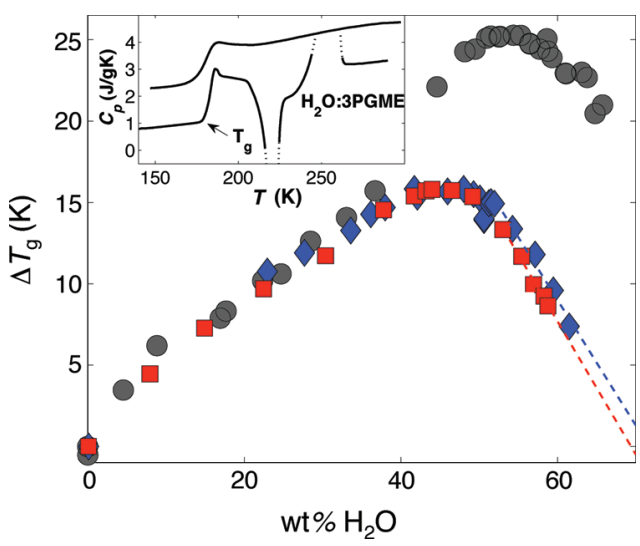

Figure 1. Difference in glass transition temperatures from that of the pure materials, $\Delta T_{\mathrm{g}}=T_{\mathrm{g}}-T_{\mathrm{g}}^{\text {pure }}$, as a function of water weight fraction for water- $n$ PGME mixtures, $n=1(\bigcirc), 2(\diamond)$, and $3(\square)$. The dashed lines are linear extrapolations for the concentration range above $\sim 50 \mathrm{wt} \%$. The inset shows the isobaric heat capacity, $C_{p}$, for a water-3PGME mixture with 55 wt \% water. The cooling data (upper data set) exhibit one distinct glass transition and no crystallization. The heating data (lower data set) display one glass transition, one crystallization and one melting event. For clarity, the cooling curve is shifted by $+1.5 \mathrm{~J} \mathrm{~g}^{-1} \mathrm{~K}^{-1}$.

contents ( $>51,40$, and $38 \mathrm{wt} \%$ for $n=1,2,3)$ one crystallization and one melting event supersedes the glass transition upon heating. We find no indications of phase separation for any of the samples. $\Delta T_{\mathrm{g}}$, the difference in $T_{\mathrm{g}}$ between the mixture and the corresponding pure $n \mathrm{PGME}\left(T_{\mathrm{g}}^{\text {pure }}=143.6,162.3,168.7 \mathrm{~K}\right.$ for $n=1,2,3)$ are shown in Figure 1. An initial growth of $\Delta T_{\mathrm{g}}$ with increasing water content is observed for all three systems, but for intermediate water fractions a maximum in $\Delta T_{\mathrm{g}}$ is reached above which $\Delta T_{\mathrm{g}}$ decreases monotonically. The results for $n=2$ and 3 are essentially identical throughout the whole concentration range, with a maximum $\Delta T_{\mathrm{g}}=16 \mathrm{~K}$ observed at 45 wt \% water (corresponding to 6.7 and 9.2 water molecules per $n$ PGME molecule, respectively). The monomeric system shows the same qualitative behavior, but here $\Delta T_{\mathrm{g}}$ shows an abrupt increase at $\sim 37$ wt $\%(\sim 75 \mathrm{~mol} \%)$ water and the maximum $\Delta T_{\mathrm{g}}=25.5 \mathrm{~K}$ takes place for $55 \mathrm{wt} \%$ water.

For binary aqueous mixtures it is not possible to cover the full concentration range, ranging from one pure material to the other, due to crystallization at high water contents. Thus, to better understand the variation over the full concentration range, we also measure the calorimetric $T_{\mathrm{g}}$ in mixtures of PGME $(n=1)$ and a $33 \mathrm{~mol} \%$ aqueous hydrogen peroxide $\left(\mathrm{H}_{2} \mathrm{O}_{2}\right)$ solution. Aqueous $\mathrm{H}_{2} \mathrm{O}_{2}$ is a good glass-former and shows strong similarities to pure water regarding boiling and melting points, permittivity, viscosity and $T_{\mathrm{g}}$ dependence upon addition of salts. ${ }^{23,24}$ We find a nonmonotonic variation of $T_{\mathrm{g}}$ with a maximum in $\Delta T_{\mathrm{g}}$ of $\sim 10 \mathrm{~K}$ around $30 \mathrm{wt} \%$ solvent, as shown in Figure 2. This nonmonotonic behavior is consistent with that observed for the mixtures of water and $n$ PGMEs. Note that we are here able to measure $T_{\mathrm{g}}$ over the full range from pure PGME to pure $33 \mathrm{~mol} \% \mathrm{H}_{2} \mathrm{O}_{2}$ /water solution. To further investigate the generality of these results, we also investigate mixtures of 3PGME and propylene glycol (PG). The latter is a H-bonded liquid with glass-transition related dynamics that have been suggested to resemble that of water. ${ }^{25}$ As shown in Figure 2, mixtures of 3PGME and PG also show nonmonotonic $T_{\mathrm{g}}$ behavior very similar to that observed for PGME and the 


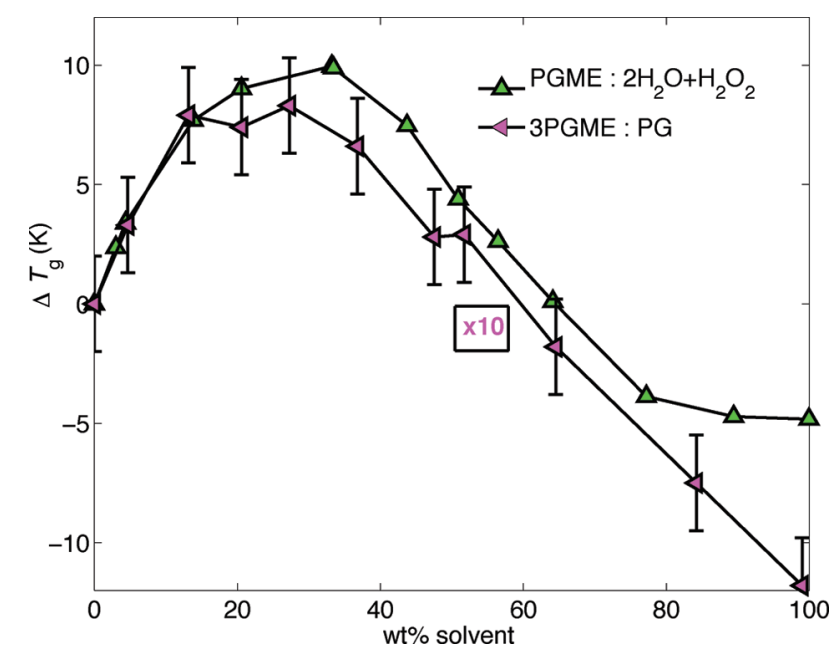

Figure 2. Difference in glass transition temperatures from that of the pure materials, $\Delta T_{\mathrm{g}}=T_{\mathrm{g}}-T_{\mathrm{g}}^{\text {pure }}$, as a function of solvent weight fraction for mixtures of PGME and $33 \mathrm{~mol} \%$ aqueous $\mathrm{H}_{2} \mathrm{O}_{2}(\Delta)$ and 3PGME and PG (triangle pointing left), respectively. For the latter system the $\Delta T_{\mathrm{g}}$ have been multiplied by a factor of 10 to facilitate an easy comparison between the two data sets.

aqueous $\mathrm{H}_{2} \mathrm{O}_{2}$ solution; the effects are, however, considerably less pronounced. Thus, we conclude that the nonmonotonic concentration variation of $T_{\mathrm{g}}$ for $\mathrm{nPGME}$ is observed both in aqueous mixtures and in mixtures of similar nature.

Returning to the aqueous $n$ PGME system, we find that for low water contents, all three $n$ PGMEs show a systematic increase of $\Delta C_{p}$ as the statistical number of water molecules per $n \mathrm{PGME}$ molecule, $X$, increases, as shown in Figure 3. A small increase in $\Delta C_{p}$ is expected for increasing $X$ only due to the increase in $T_{\mathrm{g}}$, since $\Delta C_{p}=T_{\mathrm{g}}\left[\left(\partial S^{\text {liquid }} / \partial T\right)_{p}-\left(\partial S^{\text {glass }} / \partial T\right)_{p}\right]$, where $S$ is the entropy. The effects on $\Delta C_{p}$ arising solely from this composition dependent $T_{\mathrm{g}}$ are shown in Figure 3 as dashed lines; these effects clearly give only a small contribution to $\Delta C_{p}$. Thus, the main contribution to the increase in $\Delta C_{p}$ results from a change in the $T$-dependence of the entropy at $T_{\mathrm{g}}$. For all mixtures, $\Delta C_{p}$ increases with water content up to the concentration where $T_{\mathrm{g}}$ has a maximum. These concentrations are marked by the vertical dash-dotted lines in Figure 3. A crossover in the $\Delta C_{p}$ behavior at a composition corresponding to the maximum in $\Delta T_{\mathrm{g}}$ is common to all three systems. The monomer shows a behavior qualitatively similar to that of the dimer and trimer, but consistent with its $\Delta T_{\mathrm{g}}$ behavior, a sudden increase in $\Delta C_{p}$ is observed at the corresponding concentration. For large water concentrations, $\Delta C_{p}$ level out or even decrease slightly for all three systems.

\section{DISCUSSION}

Several studies have demonstrated the important role of $\mathrm{H}$-bonding via the hydroxyl end-groups for the dynamics of pure oligomeric propylene glycols; both light scattering and dielectric relaxation studies have demonstrated polymer-like dynamics already for chains of seven repeat units, suggesting the formation of $\mathrm{H}$-bond mediated effective chains. ${ }^{25,26}$ Moreover, the glasstransition dynamics of $n$ PGMEs were compared to those of $n$ propylene glycol dimethyl ethers, $n$ PGDEs, which share the same monomeric unit but lack the hydroxyl end-groups and thus $\mathrm{H}$ bonding. The results indicate that the molecular entities characteristic of the structural relaxation are larger than the individual

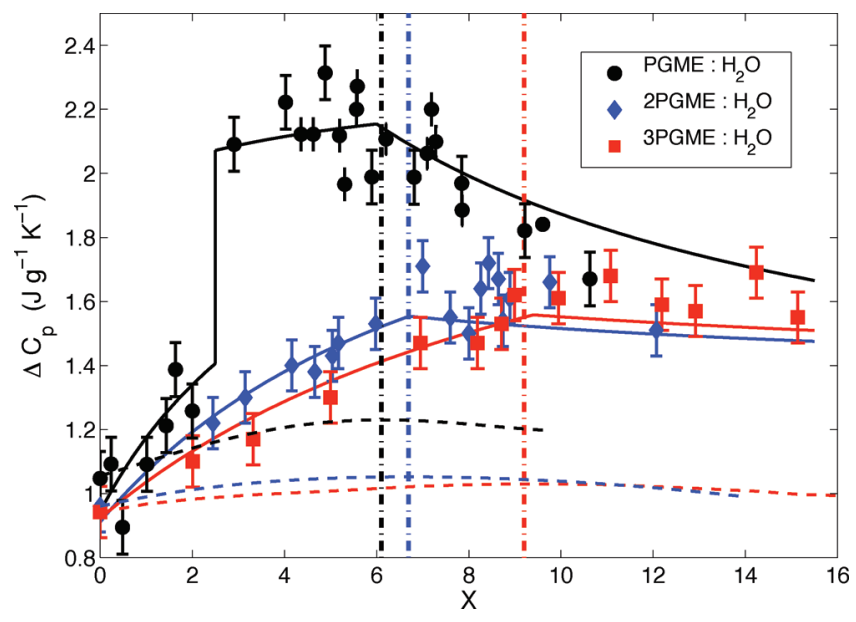

Figure 3. Heat capacity step, $\Delta C_{p}$, at $T_{\mathrm{g}}$ for $n \mathrm{PGME}$-water mixtures for $n=1$ (circles), 2 (diamonds), and 3 (squares) as a function of the water/ $n$ PGME molar ratio, $X$. The dashed lines represent the increase in $\Delta C_{p}$ only taking the increased $T_{\mathrm{g}}$ into account (see the text for details). The vertical dash-dotted lines indicate the concentrations where $T_{\mathrm{g}}$ reaches maximum values, as shown in Figure 1. The solid lines are explained in the text.

molecules meaning that effective relaxing units (ERU) are formed. The size of these ERUs were estimated and it was suggested that pure $n$ PGMEs effectively form ERUs of two molecules through an H-bond mediated coordination of hydroxyl endgroups. $^{25}$

As water is added to the $n$ PGMEs, we expect the water molecules to preferentially coordinate to the strongly $\mathrm{H}$-bonding hydroxyl end-groups, thus increasing the size of the ERUs and in turn leading to an increase in both $\Delta T_{\mathrm{g}}$ and $\Delta C_{p}$. It is interesting to note the analogous behavior for mixtures of methanol and water, where the added water form clusters that are strongly coordinated to the hydroxyl groups of the surrounding methanol molecules. ${ }^{16}$ Because of the dynamic nature of $\mathrm{H}$-bonds, we expect that an ERU saturates at some effective size, corresponding to a characteristic number of water molecules per hydroxyl group. Such a saturation should be directly manifested as a crossover in the composition dependence of both $\Delta T_{\mathrm{g}}$ and $\Delta C_{p}$. Referring to the behavior shown in Figure 1 and 2, the simplest interpretation of the maximum observed in $\Delta T_{\mathrm{g}}$ and the corresponding crossover in the temperature dependence of $\Delta C_{p}$ is thus that these are manifestations of this behavior.

To test the hypothesis that the hydroxyl $(\mathrm{OH}-)$ end-groups play a key role in the observed mixture behavior, we also study mixtures of water and tripropylene glycol dimethyl ether, 3PGDE, which has the same structure as 3PGME except that its $\mathrm{OH}$-end group has been exchanged for a methoxy group $\left(\mathrm{O}-\mathrm{CH}_{3}\right)$. For aqueous 3PGDE mixtures, single $T_{\mathrm{g}}$ solutions are formed up to concentrations $\sim 20 \mathrm{~mol} \%$ water. As shown in Figure 4, we find that $T_{\mathrm{g}}$ decreases monotonically within this concentration interval, in contrast to the behavior observed for 3PGME; this suggests a $T_{\mathrm{g}}<150 \mathrm{~K}$ for pure water. For pure water, the supercooled temperature range, $150<T<235 \mathrm{~K}^{27,28}$ is largely inaccessible due to crystallization and there is thus still no concensus regarding its $T_{\mathrm{g}}$ value. The traditional interpretation of the available data is that water has a $T_{\mathrm{g}} \sim 130-140 \mathrm{~K}$, but widely different values including $T_{\mathrm{g}} \sim 165 \mathrm{~K}^{2 \mathrm{~g}, 30}$ or $T_{\mathrm{g}} \sim 228 \mathrm{~K}^{31}$ have also been suggested. Taking into account the limited data 


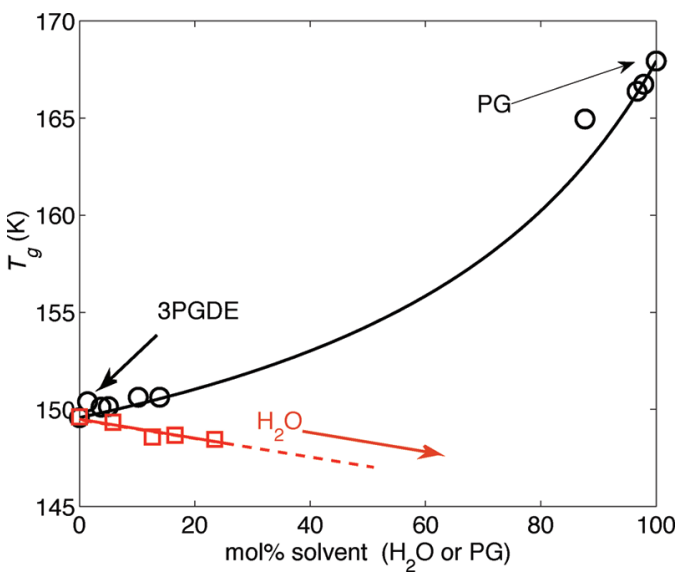

Figure 4. Composition dependent $T_{\mathrm{g}}$ for mixtures of $3 \mathrm{PGDE}$ and PG (circles) as well as 3PGDE and $\mathrm{H}_{2} \mathrm{O}$ (squares). The upper solid (black) line represents the volume weighted average between the two pure components. The red line is a guide to the eye.

range for this system, the results suggest a monotonic composition behavior with a $T_{\mathrm{g}}$ for water $\sim 140 \mathrm{~K}$; we note that this value is in agreement with results from extrapolations of $T_{\mathrm{g}}$ for dilute salt solutions. ${ }^{32,33}$ It is also interesting to note that simple linear extrapolations of the $\Delta T_{\mathrm{g}}$ data, as shown in dashed lines in Figure 1, suggest that $T_{\mathrm{g}}=141$ and $144 \mathrm{~K}$ at $100 \%$ water for the 2 and 3PGME mixtures, respectively. Moreover, a similar result with $T_{\mathrm{g}} \sim 136 \mathrm{~K}$ has been obtained from extrapolations of data for aqueous butyltripropylamonium chloride. ${ }^{14}$ However, it is important to keep in mind that the relation between the extrapolated $T_{\mathrm{g}}$ behavior for dilute solutions and that of pure bulk water is far from clear. ${ }^{31,34}$

To investigate the behavior for 3PGDE mixtures over a wider concentration range we study mixtures of $3 \mathrm{PGDE}$ and $\mathrm{PG}$, as these liquids are miscible for concentrations $<15$ or $>90 \mathrm{~mol} \%$ PG. Consistent with the aqueous mixture results, we find support for a monotonic $T_{\mathrm{g}}$ variation, which over the measured composition range is well described by the prediction for a volume weighted average of the two components. We thus conclude that the nonmonotonic effects observed for the $n$ PGME:s are directly related to the presence of the $n \mathrm{PGME} \mathrm{OH}$-group. It is interesting to compare these results with recent studies ${ }^{22,34}$ on aqueous mixtures of $n$-propylene glycols, $n \mathrm{PG}$, which share its monomeric molecular structure with $n$ PGME, but have hydroxyl groups in both ends. The chain-ends of pure $n$ PGs readily hydrogen bond, which already for the pure materials leads to the formation of extended hydrogen bonded structures. ${ }^{25,26}$ The two hydroxyl groups of $n \mathrm{PG}$ thus leads to different hydrogen bond induced structures compared with those found in $n$ PGME, for which the ERUs were estimated to be composed of 2 molecular units instead of the $\sim 8$ estimated for $n \mathrm{PG} .{ }^{25}$ Also, due to the hydrophilic nature of both its ends, $n \mathrm{PG}$ is expected to coordinate differently compared with $n$ PGME in aqueous mixtures. In agreement with this, and as demonstrated in refs 22 and 34, addition of water to $n \mathrm{PG}$ leads to a monotonic decrease of $T_{\mathrm{g}}$, which strongly contrasts the nonmonotonic concentration dependence of the $n$ PGME mixtures. We can thus conclude that the behavior observed for $n$ PGMEs is a direct result of its single hydroxyl end-group.

To quantify the role played by $\mathrm{OH}$-groups, we calculate the contribution to the heat capacity step per mole of $n$ PGME

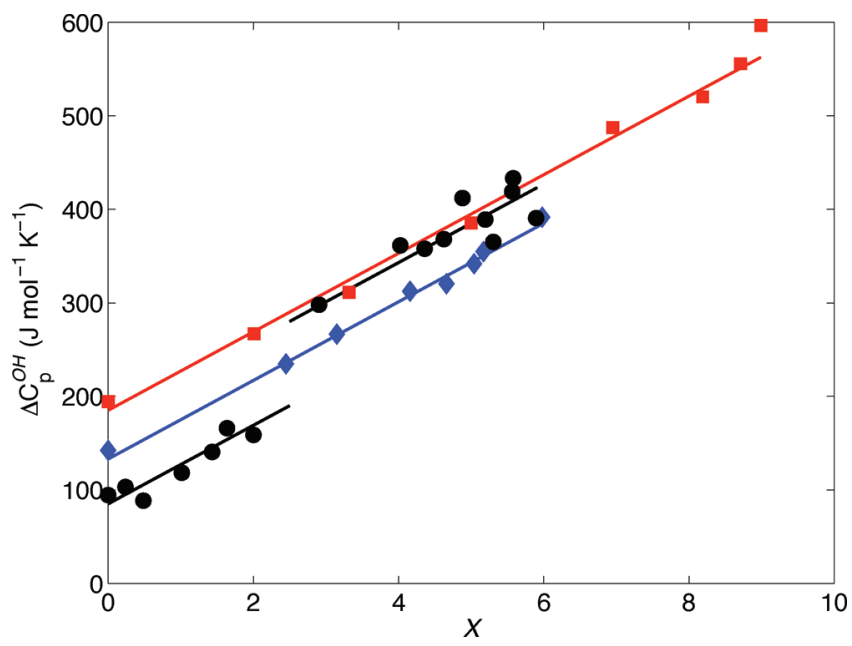

Figure 5. Amplitude of the step in the heat capacity at $T_{\mathrm{g}}$ per mole of hydroxyl groups $\Delta C_{p}{ }^{O H}$ as a function of the number of water molecules per $n$ PGME molecules for $n=1$ (circles), $n=2$ (diamonds) and $n=3$ (squares). The solid lines represent a contribution to $\Delta C_{p}$ of $41 \mathrm{~J} \mathrm{~mol}^{-1} \mathrm{~K}^{-1}$ for each water molecule statistically added per hydroxyl group.

$$
\begin{aligned}
& \text { OH-groups, } \Delta C_{p}{ }^{O H} \text { : } \\
& \qquad \begin{aligned}
\Delta C_{p}^{O H}\left[\mathrm{~J} \mathrm{~mol}^{-1} \mathrm{~K}^{-1}\right]= & \Delta C_{p}\left[\mathrm{Jg}^{-1} \mathrm{~K}^{-1}\right] \\
& \times w_{\mathrm{n}, P G M E}{ }^{-1}[\mathrm{~g} / \mathrm{g}] \\
& \times M_{\mathrm{n}, P G M E}\left[\mathrm{~g} \mathrm{~mol}^{-1}\right]
\end{aligned}
\end{aligned}
$$

where $\Delta C_{p}$ is the heat capacity per unit mass of sample, $w_{n \mathrm{PGME}}$ is the mass fraction and $M_{n \mathrm{PGME}}$ is the molar mass of $n \mathrm{PGME}$, respectively. $\Delta C_{p}{ }^{O H}$ is shown as a function of $X$, the statistical number of added water molecules per $\mathrm{OH}$-group, in Figure 5, for concentrations below saturation. For the 2- and 3PGME mixtures, we find identical linear increases of $\Delta C_{p}{ }^{O H}$ with increasing $X$, corresponding to a contribution of $\sim 41 \mathrm{~J} \mathrm{~mol}^{-1} \mathrm{~K}^{-1}$ to $\Delta C_{p}{ }^{O H}$ per added mole of water. Thus, below the saturation concentration, each water molecule statistically added per $\mathrm{OH}$ group brings a well-defined contribution to the heat capacity step. This contribution of $\sim 41 \mathrm{~J} \mathrm{~mol}^{-1} \mathrm{~K}^{-1}$ together with the $\Delta C_{p}$ values for the pure 2- and 3-PGME, respectively, predict the behavior of $\Delta C_{p}$ vs water weight fraction in the composition range below the maximum in $\Delta C_{p}$, as shown with solid lines in Figure 3. It is interesting to compare the value $41 \mathrm{~J} \mathrm{~mol}^{-1} \mathrm{~K}^{-1}$ to $\Delta C_{p}=$ $22 \pm 3 \mathrm{~J} \mathrm{~mol}^{-1} \mathrm{~K}^{-1}$, which is estimated for pure water from extrapolation of calorimetry results for dilute aqueous salt solutions. 32,33

As demonstrated in Figure 1 and 2, the behavior of the PGME monomer differs from that of the dimer and trimer by the change in both $\Delta T_{\mathrm{g}}$ and $\Delta C_{p}$ occurring for a water mole fraction $\sim 3$; this behavior is a strong indication of a structural change. Interestingly, as shown in Figure 5, we find that the slope of $\Delta C_{p}{ }^{O H}$ vs $X$ is the same before and after this change. Thus, except for the structural change observed for the monomeric system, all three systems behave in a highly similar manner, each with the same contribution to $\Delta C_{p}{ }^{O H}$ per added mole of water.

For water contents above the maximum in $\Delta C_{p}, \Delta C_{p}$ levels out or even decreases slightly, as shown in Figure 3. On the basis of the hypothesis that ERUs grow until the maximum value of $\Delta C_{p}$ is reached, we speculate that for higher water contents the excess water coexists with the saturated ERUs, acting as a plasticizer and thus resulting in a $T_{\mathrm{g}}$ decrease. As discussed above, it is here 
interesting to note that for water concentrations above those corresponding to the viscosity maximum in methanol-water mixtures, neutron diffraction studies demonstrate a percolating water phase with a local structure similar to that of pure water, ${ }^{16}$ Therefore, to describe the $\Delta C_{p}$ behavior at high water concentrations, we use a molar weighting between the literature value for pure water, $\Delta C_{p}=22 \mathrm{~J} \mathrm{~mol}^{-1} \mathrm{~K}^{-1,32,33}$ used as characteristic of the excess water, and the maximum $\Delta C_{p}$ value. The results are shown as solid lines in Figure 3, and we find that this simple approach can describe the data well.

The effects observed in this work, both for $\Delta T_{\mathrm{g}}$ and $\Delta C_{p}$, correspond to dynamics and thermodynamics acting on timescales $\sim 100$ s. If higher temperatures, or correspondingly shorter time-scales are investigated, one expects to observe less pronounced effects since it is well-known that $\mathrm{H}$-bond interactions are less effective at high temperatures. ${ }^{35}$ Consistent with this, using quasielastic neutron scattering, QENS, ${ }^{36}$ which probes time-scales of the order of $10-100 \mathrm{ps}$, a nonmonotonic variation of $\sim 5 \mathrm{~K}$ was observed for 2PGME-water mixtures ${ }^{37}$ instead of the $15 \mathrm{~K}$ observed in this work. This result is in agreement with the observed degree of nonmonotonic concentration dependence of the room temperature viscosity of water-methanol and waterethanol mixtures ${ }^{38}$ as well as the observation that the nanoscopic segregation in water-methanol mixtures is enhanced by cooling. ${ }^{39}$

\section{CONCLUDING REMARKS}

In conclusion, we here present nonmonotonic composition variations of the glass transition temperature in binary mixtures of $n$-propylene glycol monomethyl ethers ( $n$ PGMEs) and water. We demonstrate that the observed effects are directly related to the presence of $n$ PGME hydroxyl groups and show that the $T_{\mathrm{g}}$ maxima correspond to crossovers in the composition dependence of the step amplitude in the isobaric heat capacity at $T_{\mathrm{g}}$. Using a simple model based on (i) the formation of $\mathrm{H}$-bond induced effective relaxing entities and (ii) a plasticizing effect at high water contents where the relaxing entities have reached a maximum size, we account for our observations. Our results stress the importance of future work aimed at reaching a better quantitative understanding of the effects of hydrogen bond induced structure formation in binary glass forming liquids.

\section{AUTHOR INFORMATION}

\section{Corresponding Author}

*E-mail: (J.S.) johan.sjostrom@chalmers.se; (J.M.) k.j.l.mattsson@ leeds.ac.uk.

\section{ACKNOWLEDGMENT}

The work was financially supported by the Swedish Research Council, the Swedish Foundation for Strategic Research, the Knut and Alice Wallenberg Foundation (JM), and the Swedish Energy Agency.

\section{REFERENCES}

(1) Gordon, M.; Taylor, J. S. Appl. Chem. 1952, 2, 493.

(2) Cohen, M. H.; Turnbull, D. J. Chem. Phys. 1959, 34, 1164.

(3) Myers, M. B.; Felty, E. J. Mater. Res. Bull. 1967, 34, 1164.

(4) Rao, K. J.; Mohan, R. J. Phys. Chem. 1980, 84, 1917.

(5) Zielniok, D.; Cramer, C.; Eckert, H. Chem. Mater. 2007, 19, 3162.

(6) Lesikar, A. V. J. Chem. Phys. 1977, 66, 4263.
(7) Wang, L. M.; Richert, R. J. Phys. Chem. B 2005, 109, 11091.

(8) Takeda, K.; Murata, K.; Yamashita, S. J. Phys. Chem. B 1999, $103,3457$.

(9) Wang, L. M.; Tian, Y.; Liu, R.; Richert., R. J. Phys. Chem. B 2010, 114, 369.

(10) Amines can donate two and accept one H-bond, whereas alcohols can donate one and accept two. Thus, the maximum number of H-bonds form at an equimolar concentration, which in turn corresponds to a maximum in $T_{g}$.

(11) Nagahama, M.; Suga, H.; Andersson, O. Thermochim. Acta 2000, 363, 1657.

(12) Prestrelski, S. J.; Tedeschi, N.; Arakawa, T.; Carpenter, J. F. Biophys. J. 1993, 65, 661.

(13) Ohnishi, A.; Kanno, H. Chem. Phys. Lett. 1996, 263, 259.

(14) Kanno, H.; Yokoyama, H.; Yoshimura, Y. J. Phys. Chem. B 2001, 105, 2019.

(15) Frank, H.; Evans, M. J. Chem. Phys. 1945, 13, 507.

(16) Dixit, S.; Crain, J.; Poon, W. C. K.; Finney, J. L.; Soper, A. K. Nature 2002, 416, 829.

(17) Soper, A. K.; Dougan, L. J. Phys. Chem. B 2006, 110, 3472.

(18) Dougan, L.; Bates, S. P.; Hargreaves, R.; Fox, J. P.; Crain, J.; Finney, J. L.; Réat, V.; Soper, A. K. Jour Chem. Phys. 2004, 121, 6456.

(19) Srinivasan, A.; Bermejo, F. J.; de Andres, A.; Dawidowski, J.; Zuniga, J.; Criado, A. Phys. Rev. B 1996, 53, 8172.

(20) Murthy, S. S. N. J. Phys. Chem. A 1999, 103, 7927.

(21) Sjöström, J.; Bergman, R.; Swenson, J.; Wadell, C.; Moberg, T.; Mattsson, J. J. Phys. Chem. B 2011, 115, 1842.

(22) Sjöström, J.; Mattsson, J.; Bergman, R.; Johansson, E.; Josefsson, K.; Svantesson, D.; Swenson, J. Phys. Chem. Chem. Phys. 2010, 12, 10452.

(23) Ghormley, J. A. J. Am. Chem. Soc. 1957, 79, 1862.

(24) Minoguchi, A.; Richert, R.; Angell, C. A. Phys. Rev. Lett. 2004, 93, 215703.

(25) Mattsson, J.; Bergman, R.; Jacobsson, P.; Börjesson, L. Phys. Rev. B 2009, 79, 174205.

(26) Bergman, R.; Svanberg, C.; Andersson, D.; Brodin, A.; Torell, L. M. J. Non-Cryst. Solids 1998, 235-237, 225.

(27) Johari, G. P.; Hallbrucker, A.; Meyer, E. Nature 1987, 330, 552.

(28) Hallbrucker, A.; Meyer, E.; Johari, G. P. J. Phys. Chem. 1989, 93, 4986.

(29) Ito, K.; Moynihan, C. T.; Angell, C. A. Nature 1999, 398, 492.

(30) Cerveny, S.; Schwartz, G. A.; Bergman, R.; Swenson, J. Phys. Rev. Lett. 2004, 93, 245702.

(31) Swenson, J.; Teixeira, J. J. Chem. Phys. 2010, 132, 014508.

(32) Angell, C. A.; Sare, E. J. J. Chem. Phys. 1970, 52, 1058.

(33) Angell, C. A.; Tucker, J. C. J. Phys. Chem. 1980, 84, 268.

(34) Cerveny, S.; Schwartz, G.; Alegria, A.; Bergman, R.; Swenson, J. J. Chem. Phys. 2006, 124, 194501.

(35) Walrafen, G. E. J. Chem. Phys. 1967, 47, 114

(36) Swenson, J.; Sjöström, J.; Fernandez-Alonso, F. J. Chem. Phys. 2010, 133, 234506.

(37) This is estimated from the composition dependent variation of the relaxation time at $280 \mathrm{~K}$ and $Q=0.7 \AA^{-1}$.

(38) Ageno, M.; Frontali, C. Proc. Natl. Acad. Sci. U.S.A. 1967, 57, 856.

(39) Dougan, L.; Hargreaves, R.; Bates, S. P.; Finney, J. L.; Réat, V.; Soper, A. K.; Crain, J. J. Chem. Phys. 2005, 122, 174514. 